\title{
La diversité des camps d'étrangers en Europe : présentation de la carte des camps de Migreurop
}

Caroline Intrand et Pierre-Arnaud Perrouty

\section{(2) OpenEdition \\ 1 Journals}

\section{Édition électronique}

URL : http://journals.openedition.org/conflits/1727

DOI : $10.4000 /$ conflits. 1727

ISSN : $1777-5345$

Éditeur :

CCLS - Centre d'études sur les conflits lilberté et sécurité, L'Harmattan

\section{Édition imprimée}

Date de publication : 1 mars 2005

Pagination : 71-90

ISBN : 2-7475-8103-9

ISSN : 1157-996X

\section{Référence électronique}

Caroline Intrand et Pierre-Arnaud Perrouty, « La diversité des camps d'étrangers en Europe: présentation de la carte des camps de Migreurop », Cultures \& Conflits [En ligne], 57 | printemps 2005, mis en ligne le 06 janvier 2010, consulté le 30 mars 2021. URL : http://journals.openedition.org/ conflits/1727 ; DOI : https://doi.org/10.4000/conflits.1727

Ce document a été généré automatiquement le 30 mars 2021.

Creative Commons License 


\title{
La diversité des camps d'étrangers en Europe : présentation de la carte des camps de Migreurop
}

\author{
Caroline Intrand et Pierre-Arnaud Perrouty
}

1 Depuis fin 2001, le réseau Migreurop rassemble chercheurs, élus et militants associatifs de plusieurs pays européens pour la collecte, l'analyse et la diffusion d'informations relatives aux politiques européennes d'asile et d'immigration qui mènent à la relégation d'étrangers dans des camps.

2 Le réseau Migreurop est né d'une initiative collective après le constat que le centre de Sangatte, lieu de regroupement de centaines de migrants cherchant à gagner le Royaume-Uni géré par la Croix Rouge jusqu'à fin 2003, n'était autre que le symbole d'une politique d'asile et d'immigration défaillante puisqu'elle aboutissait à rassembler des populations sans statut juridique dans des lieux hors droit. Avec le concours d'ONG de terrain et de chercheurs, le réseau s'est ainsi lancé dans l'analyse d'informations et de données sur les lieux de regroupement de migrants à travers l'Europe pour aboutir à une définition et une typologie des camps. Ces recherches ont ensuite permis l'élaboration de la "carte des camps d'étrangers en Europe $»^{1}$ (dont la dernière version fait également apparaître certains lieux en Afrique du Nord). Cette carte, qui pour des raisons pratiques représente les lieux fermés et identifiables auxquels ont été ajoutés certains lieux ouverts caractéristiques, permet de visualiser l'ampleur du phénomène de mise à distance des étrangers en Europe.

Mais la notion de camp d'étrangers pour Migreurop n'est pas uniquement définie par les lieux de privation de liberté tels que les camps de rétention, de détention ou les prisons $^{2}$. Afin de ne pas occulter une partie de la réalité des conséquences des politiques migratoires en Europe, le réseau a étendu le concept de « camps » à tout lieu de relégation où l'étranger est privé de ses droits - parfois seulement partiellement. Le terme va donc définir tous les types de lieux de mise à distance des étrangers : ainsi certains centres ouverts d'accueil, de transit ou d'hébergement, dans lesquels les étrangers sont cantonnés parce qu'ils n'ont pas d'autre choix que celui de s'y trouver, 
seront entendus comme des camps. Les lieux de regroupement informels des étrangers en errance, ou de regroupement dans l'attente du passage clandestin d'une frontière, comme en son temps Sangatte ou actuellement les camps du nord du Maroc, font également partie de ce que Migreurop rassemble sous le mot camp.

Toutes les techniques de mise à l'écart, du maintien à distance et la politique des visas à l'expulsion en passant par la notion « d'externalisation » qui se dessine de plus en plus nettement dans le paysage européen font également partie de l'objet d'étude de Migreurop dans la mesure où ce sont des politiques créatrices de camps.

Les recherches de Migreurop - et c'est également l'objet du présent article - ont abouti à faire émerger la grande diversité de ces camps, de par leurs régimes, leurs fonctions, leur configuration spatiale... Elles ont également eu pour objectif de montrer leur caractéristiques communes : leurs occupants - étrangers n'ayant commis aucun délit en dehors de celui du franchissement illégal d'une frontière -, la disparition de l'individu au profit du groupe et la difficulté d'y assurer le respect des droits fondamentaux. Ces traits dominants illustrent les conséquences des politiques européennes d'asile et d'immigration qui utilisent les camps comme outil de gestion des migrations. Les tous récents projets de camps ou « centres d'accueil » dans les pays frontaliers à l'extérieur de l'Union ne sont qu'un élément de plus qui confirme la logique européenne.

\section{Typologie des lieux de mise à l'écart}

6 Tous les Etats de l'Union européenne procèdent, sous une forme ou une autre, à la mise à l'écart des étrangers trouvés sans titre de séjour sur leur territoire. La première image qu'évoque le terme "camp», c'est celle d'un lieu fermé, physiquement identifiable et localisable, dont la fonction première est de maintenir sous contrôle des personnes indésirables. Présents en grand nombre sur le territoire de l'Union, ces camps prennent des formes multiples : camps ouverts, fermés, construits ad hoc pour l'enfermement en vue de l'expulsion, anciens bâtiments réaffectés durablement ou réquisitionnés dans l'urgence pour faire face à une crise, prisons, locaux divers. Des camps de fortune improvisés dans les îles grecques ou au sud de l'Italie aux centres de rétention français ou aux centres fermés belges, des prisons allemandes aux campstampons des enclaves de Ceuta et Melilla au Maroc, la réalité des camps en Europe est donc avant tout multiforme.

7 Toute tentative de typologie est dès lors délicate, d'autant que cette figure classique du camp, entité physique localisable, rend mal compte de la diversité des dispositifs administratifs et policiers déployés pour maintenir à l'écart ceux qui tentent de gagner l'Europe. Politique de délivrance des visas, opacité des décisions administratives, découragements sous des formes diverses, contrôles aux frontières, maintien en zone de transit des aéroports, regroupement dans des centres "ouverts" aux fins de contrôle, les barrières ne manquent pas. En ce sens, le camp ne recouvre pas seulement des lieux identifiables mais l'ensemble de processus et de dispositifs qui visent à maintenir à l'écart, à rendre invisible et à éloigner. Il reste que le caractère ouvert ou fermé des camps parait être la distinction de départ la plus claire et la plus intuitive. 


\section{Lieux ouverts}

8 Ces lieux ont pour objectif premier d'enregistrer les migrants, de surveiller leurs activités et, surtout, de pouvoir les localiser facilement dans l'hypothèse - tellement souvent vérifiée - où il faudrait exécuter une décision d'éloignement. En d'autres termes : opérer un contrôle administratif et social sur les migrants.

9 Ces lieux sont, eux aussi, multiples. Ils peuvent prendre la forme de centres d'accueil pour demandeurs d'asile où les candidats au statut de réfugié sont nourris et logés. La Belgique, par exemple, conditionne l'octroi de l'aide sociale en nature au fait de rester dans ces centres. Mais ces lieux peuvent aussi prendre des formes moins visibles, comme l'obligation de se présenter régulièrement à un guichet de l'administration chargée du traitement de leur dossier. Il n'est pas rare que le fait de ne pas répondre à une de ces convocations entraîne automatiquement un rejet de la demande l'administration belge parle de « refus technique » dans ce cas. Des regroupements plus informels encore se créent régulièrement en fonction d'une situation spécifique, sous le contrôle parfois discret des autorités. On pense bien sûr au cas de Sangatte qui, de ce point de vue aussi, revêt un caractère exemplaire. Ouvert par les autorités françaises en 1999, ce camp devait héberger des migrants dépourvus de documents de séjour ou de visas qui, arrivés dans le Pas-de-Calais dans l'espoir de franchir la Manche pour se rendre en Grande-Bretagne, s'y trouvaient bloqués en raison du contrôle exercé par ce pays - non signataire de la Convention de Schengen - pour le franchissement de sa frontière. De regroupements informels dans et aux abords de la ville de Calais, on est passé à l'établissement d'un camp dans l'urgence dont la gestion a été confiée à la Croix Rouge pour parer à une situation humanitaire dramatique. Camp ouvert, certes, mais très surveillé par la police dès le départ et dont les entrées et sorties sont devenues de plus en plus contrôlées avec le temps. A défaut de vision politique cohérente, la gestion de cette situation s'est faite sous un angle humanitaire et policier. La fermeture du camp à la fin de l'année 2002 n'a d'ailleurs que déplacé le problème vers d'autres villes côtières. Aujourd'hui encore, les conséquences de la fermeture se font sentir dans les bois aux alentours de Calais où se créent des campements de fortune, lieux parfaitement connus des forces de l'ordre qui passent régulièrement contrôler - et déloger - ceux qui y ont trouvé refuge. Conséquences parfois plus éloignées géographiquement: un certain nombre de migrants qui comptaient se rendre à Sangatte ont ainsi été bloqués à la gare du Nord à Paris suite au renforcement des contrôles et ont été contraints d'investir un certain nombre de lieux aux alentours, principalement dans le $X^{\text {ème }}$ arrondissement. Or l'exemple de Sangatte n'est pas un cas isolé : des regroupements informels continuent d'apparaître ici ou là, à la faveur d'une situation particulière, d'un lieu stratégique sur les routes migratoires ou d'un lieu à l'abandon. Comme dans cette gare désaffectée à Rome, rebaptisée "Hôtel Africa ", où des migrants, essentiellement sub-sahariens, ont élu domicile dans l'attente d'une hypothétique régularisation.

Si ces regroupements se produisent dans des conditions difficiles, les conditions se dégradent encore lorsqu'ils sont situés aux abords des frontières de l'Union. On pense par exemple aux campements marocains dans les forêts de Bel Younech ou de Gourougou ou aux campements de fortune de Maghnia en Algérie, près de la frontière marocaine. Les migrants s'y regroupent, vivent dans des conditions sanitaires déplorables et se trouvent à la merci des forces de l'ordre locales. Au mois d'avril 2004, 
la police de Tetouan au Maroc menait une vaste opération de ratissage dans le camp de Bel Younech. Une association marocaine rapporte que les policiers ont ainsi confisqué près de quatre tonnes d'aide humanitaire, trois cents couvertures et procédé à de nombreuses arrestations ${ }^{3}$.

\section{Lieux fermés}

11 Si on s'attache aux camps fermés, le plus pertinent semble être de les classer selon la fonction qui leur est assignée. On peut globalement en distinguer trois types, en fonction de la situation administrative des personnes qui s'y trouvent.

Le premier type regroupe les camps d'attente où les personnes sont détenues dans l'attente de leur identification, d'un examen de leur situation et d'une éventuelle admission sur le territoire. Les camps d'attente sont situés à proximité immédiate du point de passage d'une frontière: aéroports, gares et ports. Les zones d'attente en France remplissent cette fonction, de même que les holding centers anglais ou le centre de transit de l'aéroport de Bruxelles. Les personnes qui ne sont pas admises sur le territoire seront directement refoulées dans leur pays ou dans le pays dont elles viennent, sans jamais avoir eu formellement accès au territoire. Du point de vue de certains Etats ${ }^{4}$, les personnes qui sont maintenues dans ces zones ne sont pas détenues : elles sont libres de repartir pour leur pays ou pour tout autre Etat qui les accepte. En d'autres termes: "vous pouvez aller partout, mais pas chez nous», le "nous » devant s'entendre au sens de l'Espace Schengen - illustration du célèbre principe « not in my backyard».

13 L'autre enjeu de ces zones d'attente est économique. Si du point de vue de la personne détenue, il importe peu qu'elle le soit dans un centre réputé «à la frontière » ou sur le territoire, la distinction est importante du point de vue de l'Etat. La Convention de Chicago du 7 décembre 1944 relative à l'aviation civile internationale oblige une compagnie aérienne à reprendre à ses frais les personnes qu'elle a transportées et qui sont refoulées faute de documents. Obligation qui cesse dès que ces personnes ont été admises sur le territoire. En Belgique par exemple, l'Etat facture aux compagnies aériennes les frais de séjour et d'éloignement des migrants qu'elles ont transportés. Or, faire porter la charge financière des refoulements sur les transporteurs entraîne une certaine confusion des rôles qui peut mener à des dérives inquiétantes. La défunte Sabena, compagnie nationale belge, avait ainsi conclu un protocole d'accord avec le ministre de l'Intérieur le 24 mai 2000. Cet accord prévoyait l'organisation de refoulements avec une escorte constituée du personnel de sécurité de la Sabena. C'est seulement en cas de difficultés particulières que la compagnie aérienne pouvait faire appel au détachement spécialisé de la police fédérale. Ce protocole d'accord autorisait la compagnie à avoir des contacts avec les personnes à refouler en vue de les "préparer au départ». L'actuelle $S N$ Brussels Airlines a repris cet accord à son compte: des témoignages, confirmés par des membres de l'administration, indiquent que des agents de la SN Brussels Airlines se rendent dans les centres fermés pour tenter de convaincre les personnes de repartir par leurs lignes. Avec, à la clé, des incitations diverses (généralement une somme d'argent ou l'offre de billets sur des lignes intérieures du pays de destination) et, au besoin, des menaces explicites : "Si vous ne partez pas avec nous, ce sera par avion militaire!", avions militaires loués par le ministère de l'Intérieur à celui de la Défense et dont le coût sera évidemment facturé à la compagnie aérienne. 
14 Les camps de détention constituent le deuxième type: ils visent des personnes déjà entrées sur le territoire. Les centres de détention en Grande-Bretagne répondent à cette fonction, de même que les centres d'internement pour étrangers en Espagne. Ces lieux sont aussi destinés à détenir des demandeurs d'asile en cours de procédure qui sont soupçonnés de vouloir s'évanouir dans la nature avant l'issue de la procédure.

Enfin, troisième type, les camps d'éloignement où les personnes sont détenues en vue de leur expulsion du territoire. C'est le cas des centres de rétention en France, des centres fermés en Belgique ou des centres de détention en Allemagne.

16 Si cette typologie présente le mérite d'attirer l'attention sur les différentes fonctions des camps, il faut garder à l'esprit que la plupart des camps en Europe cumulent au moins deux fonctions et se situent donc à cheval sur plusieurs types. C'est notamment le cas des centres de séjour temporaire en Italie, des camps espagnols, des centres de rétention français (qui cumulent les fonctions de détention et d'éloignement), des centres fermés belges et de la plupart des centres anglais, italiens ou polonais.

17 A l'inverse, certains lieux fermés s'accommodent mal de cette typologie parce qu'ils sont par exemple initialement prévus pour un autre usage. C'est le cas des prisons qui sont utilisées dans plusieurs pays pour détenir des migrants qui n'ont commis que le seul délit de franchissement illégal d'une frontière. L'Allemagne y recourt largement. Des ailes spéciales sont parfois aménagées mais, le plus souvent, les migrants côtoient les détenus de droit commun. En Belgique, les prisons sont utilisées quand il n'y a plus de places disponibles dans les centres. Dans certains cas il s'agit de lieux informels, pas ou peu visibles, dont il n'existe le plus souvent pas de liste officielle. C'est le cas des locaux de rétention en France que chaque préfecture peut créer par simple arrêté en fonction de ses besoins. Commissariats de police, gendarmeries ou chambres d'hôtel réquisitionnées, on peut dénombrer près d'une centaine de ces lieux sur le territoire. Les conditions matérielles de détention y sont très variables et l'exercice de leurs droits est loin d'être garanti aux migrants qui y sont retenus.

\section{Limites de la distinction}

18 Si la distinction entre centres ouverts et fermés est apparemment cruciale du point de vue des personnes qui s'y trouvent, la réalité est à nuancer. D'une part, parce que les contraintes administratives et les règles internes de fonctionnement des centres ouverts peuvent aboutir à limiter sérieusement la liberté de circulation des migrants. Par exemple, en Allemagne, les migrants placés dans les Ausreizencentrum (centres « de départ») peuvent circuler dans la ville attenante au centre mais ne peuvent s'aventurer au-delà d'une certaine limite géographique. Dans certains centres ouverts en Belgique, des règles de couvre-feu imposent aux personnes de rentrer avant une certaine heure. D'autre part, parce que les centres ouverts procèdent en réalité d'une même logique de contrôle et de mise à l'écart que les centres fermés. Il s'agit de garder le contrôle sur des personnes potentiellement éloignables dans un avenir proche. En Belgique, le gouvernement procède à des éloignements au départ de centres ouverts. Ainsi, ni du point de vue de l'Etat, ni même de celui des personnes concernées, la distinction entre centres ouverts et fermés n'est totalement pertinente. 


\section{Diversité des régimes}

\section{L'encadrement juridique de la détention et de la mise à l'écart}

19 Lorsqu'il existe des normes juridiques encadrant la détention, elles permettent de faciliter le traitement des étrangers en situation administrative incertaine: identification, dissuasion, éloignement. Ces normes ont également pour objectif de légitimer des pratiques et parfois de donner l'illusionde répondre aux exigences du droit international en matière de protection des droits de l'Homme. L'histoire de l'Europe est jalonnée de lieux d'enfermement pour étrangers généralement justifiés par un état d'exception, comme c'est le cas de la France depuis le début du siècle avec l'internement des Allemands et des Autrichiens présents sur son territoire puis les camps de 1939. L'apparition de normes juridiques encadrant la détention des étrangers en Europe est néanmoins beaucoup plus récente que les pratiques de mise à l'écart.

Seuls les Etats d'immigration traditionnels ont commencé à légaliser la détention dans les années 1970. La détention des étrangers apparaît dans les lois britanniques sous le fameux " power to detain » au travers du 1971 Immigration Act $^{5}$. Il a fallut près de six ans, après que le scandale du centre de rétention d'Arenc ait été révélé au grand public en 1975, pour que la France légifère sur la rétention : la loi Bonnet du 10 janvier 1980 créé le principe de l'enfermement administratif des étrangers non admis à l'entrée sur le territoire puis la loi Questiaux ajoute à l'ordonnance du 2 novembre 1945 l'article 35 bis qui régit encore le maintien des étrangers éloignés dans des «locaux ne relevant pas de l'administration pénitentiaire ».

21 A la fin des années 1990, des semblants de législations sur la détention fleurissent dans la plupart des pays européens: l'Italie crée les Centri de Permanenza Temporanea e Assistenzas (CPTA) par une loi de $1998^{6}$ permettant la détention de toute personne entrée irrégulièrement sur le territoire national et dont l'identité n'est pas établie et de tout demandeur d'asile débouté et sous le coup d'un éloignement administratif. Dans les nouveaux pays européens, devenant potentiellement pays d'immigration, des normes ont été introduites en réponse aux exigences européennes sur l'asile et sur la répression aux frontières. A Malte par exemple, le Refugee Act de 2000 prévoit que le "Ministre responsable de l'immigration peut placer les demandeurs d'asile dans des lieux qu'il désigne et cela dans certaines circonstances $»^{7}$, sans préciser lesquelles. La Slovénie, bon élève de l'Union européenne, s'est dotée au début des années 2000 d'une législation sur les étrangers qui prévoit notamment la possibilité de détention des demandeurs d'asile durant la procédure et qui garantit un certain nombre de droits (accès à un interprète, à un avocat, à l'aide juridictionnelle...).

Issues de traditions juridiques fort différentes, les normes sur la détention ont en commun de donner des limites à l'exceptionnalisme et de garantir sur le papier des droits à ceux à qui l'on refuse en réalité toute légitimité politique et juridique. Ces normes, couvrant l'Etat de droit dans des pratiques peu avouables, sont conçues pour laisser la marge de manœuvre la plus importante possible aux autorités pour décider qui maintenir, combien de temps et où.

Les camps «informels» échappent totalement à cet encadrement: le centre de Sangatte en est l'illustration la plus connue. Les «squats » de Tiburtina à Rome ou de Cachan près de Paris sont autant de lieux de regroupement où se sont massés spontanément des centaines d'étrangers en situation précaire dans l'attente d'une 
évolution de leurs dossiers administratifs. En dehors des dispositions sur l'utilisation de locaux vides, ces lieux de regroupement échappent à tout cadre juridique particulier qui pourrait concerner l'accueil des demandeurs d'asile ou de migrants.

\section{Les détenus : sujets de droit?}

24 Ces différentes législations ont pour objet de garantir un certain nombre de droits aux détenus : droit à un recours contre la décision de refus du statut de réfugié, contre la décision d'éloignement, contre la décision de placement en détention; possibilité de faire appel à un avocat ou un conseil juridique; assistance d'un interprète et d'un médecin; droit de visite et de communication avec l'extérieur... Mais l'exercice pratique de ces droits dans les centres fermés est soumis à un fort aléa et cela, quel que soit le degré d'encadrement des systèmes. Cet aléa repose sur l'imprécision et la complexité des législations qui laissent une grande marge de manœuvre à l'administration, et également sur le manque de volonté de reconnaître les détenus comme sujets de droit.

Tout d'abord, les possibilités de recours contre l'enfermement ne sont pas toujours prévues pour être effectives : en Grande-Bretagne, il n'existe pas de contrôle de la légalité du placement par une autorité judiciaire indépendante. Les seuls moyens de recours sont les procédures de droit commun qui reviennent à demander la libération de la personne en invoquant la « clémence » de l'administration (habeas corpus, judicial review) ou en passant par la liberté sous caution (bail). Bien entendu, ces procédures sont très lentes et très chères. En Allemagne, les recours ne sont pas suspensifs et les tribunaux n'ont pas de délais pour rendre leurs jugements.

L'ineffectivité est d'autant plus grande que l'accès à l'information est réduit : en France et en Belgique par exemple ${ }^{8}$, l'absence régulière d'interprètes ne permet pas aux personnes détenues de savoir même où elles se trouvent, pourquoi et pour combien de temps. Cet accès à l'information est soumis au bon vouloir des interlocuteurs présents dans le centre : garde policière ou privée, personnel de gestion, médecins et assistants sociaux, dont le rôle est de faire "tourner la machine ", non de tenir les personnes informées de leur droits et de leur sort.

En dehors de la France, qui depuis 20 ans a laissé entrer une organisation indépendante dans les centres de rétention administrative pour l'aide à "l'exercice effectif des droits ", les détenus en Europe n'ont pas véritablement accès à un conseil juridique indépendant en dehors des potentielles visites d'avocats, de « visiteurs » appartenant à des $\mathrm{ONG}^{10}$, ou de visites pastorales. Et les avocats dans de nombreux pays se plaignent de la difficulté de travailler pour les détenus ${ }^{11}$ : petits revenus (et accès rare à l'aide juridictionnelle), difficultés d'accès aux centres en raison de leur éloignement géographique ou de leurs horaires de visite, défaut d'interprète... Il arrive donc que des migrants soient détenus de longs mois voire expulsés sans avoir pu s'entretenir avec un avocat.

\section{La durée de la détention}

Les durées de détention varient beaucoup d'un pays à un autre et certaines peuvent être extrêmement longues. Symbolique de la fermeté des systèmes et de la puissance des Etats, une longue durée de détention participe d'un moyen de dissuasion et d'écrasement des individus. Comptée dans la plupart des pays en mois plutôt qu'en 
jours ou en semaines, la durée de la détention n'a juridiquement pas de limite dans certains pays, ce qui permet la détention pendant des années de personnes qui n'ont fait qu'enfreindre la législation sur les étrangers. C'est le cas de la Grande-Bretagne, qui a échappé à une condamnation par la Cour Européenne des Droits de l'Homme dans l'affaire Chahal, malgré une détention de près de $2 \mathrm{ans}^{12}$. C'est le cas également à Malte où des personnes ont été détenues plus de 18 mois dans le centre de Floriana. L'Allemagne limite la détention à 18 mois, suivie de près par la Pologne où l'on peut détenir au maximum pendant un an. La Belgique autorise une détention qui peut aller jusqu'à 8 mois mais il n'est pas rare de rencontrer des personnes détenues depuis plus de 10 mois. En Slovénie, Slovaquie et République tchèque, la détention est limitée à 6 mois.

Les pays du Sud, à l'exception de Malte et de la Grèce ( 3 mois maximum mais en pratique, la durée peut dépasser largement cette limite), ont adopté des durées de détention moins longues. En France, le maintien en zone d'attente dure au maximum 20 jours, délai au-delà duquel la personne doit être remise en liberté. Le délai de rétention a triplé en 2003 passant de 12 à 32 jours. Objectif affiché : laisser du temps à l'administration pour l'organisation des expulsions, notamment pour l'obtention des laissez-passer consulaires indispensables à la reconduite des personnes dépourvues de document d'identité. L'Espagne se « limite » à 40 jours de détention, et l'Italie à 60 jours depuis que la loi Bossi-Fini ${ }^{13}$ a doublé cette durée.

Les durées moyennes de détention varient tout autant: en France on avoisine actuellement les 10 jours, 26 à 32 jours en Belgique, 1 à 4 mois dans $65 \%$ des cas en Grande-Bretagne.

\section{Les conditions de détention}

31 Dans les pays où les durées de détention sont très longues, les conditions sont généralement " correctement inhumaines $~^{14}$ : malgré les conditions de garde strictes, les détenus ont accès à des facilités telles que salles de sport, ateliers artistiques et bibliothèques... On y organise des fêtes, des barbecues, des compétitions sportives et occasionnellement des concerts, ce qui renforce le sentiment carcéral car par ailleurs les sorties sont minutées, les sanctions sévères et les détenus appelés par leur matricule.

En revanche, là où la détention est plus courte, les conditions sont souvent beaucoup plus précaires. Les organisations non gouvernementales qui ont pu pénétrer dans certains lieux témoignent et dénoncent: en Italie, Médecins Sans Frontière fait longuement état dans son rapport ${ }^{15} \mathrm{~d}^{\prime}$ une insuffisance grave en matière $\mathrm{d}^{\prime}$ hygiène et d'un manque de matériel médical dans la plupart des centres. Cette dénonciation a valu à l'organisation humanitaire une interdiction d'accès aux CPTA. Les conditions de détention dans le centre de Las Palmas dans les Iles Canaries ont fait l'objet d'un rapport de Human Rights Watch.

L'organisation Human Rights Watch qui a visité les centres de détention des Iles Canaries (Fuerteventura et Lanzarote) en Espagne en 2002 rapporte que les conditions de détention sont inférieures aux normes fixées par la législation nationale espagnole et le droit international : isolement total, pas de téléphone ni de visite, les détenus ne peuvent quitter les bâtiments, ni faire de l'exercice ; les médecins bénévoles ont arrêté en 2002 d'y travailler pour dénoncer les conditions sanitaires jugées " très préoccupantes $\aleph^{16}$. Dans la majorité des cas, cette précarité est accentuée par la 
surpopulation. Le CPTA de Lampedusa a récemment accueilli plus de 1300 personnes pour une capacité de 180 places.

Dans certains lieux, les détenus sont soumis à la fois à la précarité, la surpopulation et une longue durée de détention : pour Malte, le récent rapport d'enquête de la Fédération Internationale des Droits de l'Homme fait état d'une " préoccupante densité de population » et de l'inadaptation évidente des structures à la fonction de détention de longue durée ${ }^{17}$. En Grèce et de manière anecdotique, ulcérés par les conditions d'entassement des migrants arrivant des côtes turques dans la caserne désaffectée qui sert de centre de détention, les agents de la police portuaire et les policiers de l'île de Lesbos ont manifesté pour la construction d'un centre de rétention remplissant «des conditions de sécurité décentes pour ceux qui y travaillent et des conditions d'hygiène pour les étrangers ${ }^{18}$.

Les conditions sanitaires sont aussi liées au degré de visibilité des lieux de mise à l'écart. Les camps les plus inaccessibles, fermés ou informels sont évidemment ceux dans lesquels elles sont les plus inacceptables. Les camps informels du Maroc déjà évoqués, cachés au fond des forêts de Gourougou ou de Bel Younech, autour des enclaves espagnoles de Ceuta et de Melilla sont des lieux où les personnes, rejetées par une majeure partie de la population avoisinante, pourchassées par les autorités marocaines, vivent sans aucune ressource, se nourrissent dans les décharges publiques et se soignent « entre-eux ». Plusieurs personnes y sont décédées faute de soins ${ }^{19}$.

\section{Traits communs des lieux d'enfermement}

Au-delà de leur diversité fonctionnelle et de la disparité de leurs régimes, les camps d'étrangers présentent d'importants traits communs.

Tout d'abord, premier trait commun, ces lieux trahissent et entretiennent une vision totalement déshumanisée des migrants. Ils se voient le plus souvent attribuer un numéro de matricule - les noms étrangers sont notoirement difficiles à prononcer, bien que cette manière de procéder soit assez caractéristique des lieux d'enfermement en général - sont classés en catégories - la Belgique en connaît essentiellement deux: demandeur d'asile ou illégal - et identifiés à toutes les personnes de la même nationalité, au mépris des trajectoires individuelles. Bien que les régimes varient, la vie de groupe est très présente dans les camps avec son lot de difficultés de coexistence et de tensions inévitables entre des personnes en situation de détresse et aux habitudes culturelles très diverses. A cela s'ajoutent une surveillance étroite et une prise en charge totale des détenus par les gardiens, sources de vexations quotidiennes ${ }^{20}$. Dans une lettre collective, des détenus du centre fermé de Merksplas en Belgique témoignaient: "Notre dignité d'être humain est touchée: nous sommes comptés à chaque porte, chaque sortie et rentrée, il est interdit de dormir ou de faire une petite sieste, on nous interdit sans explication tout ce qui est humain, il ne reste plus qu'à nous mettre des boucles d'oreilles avec des numéros, nous sommes devenus des numéros, plus des êtres humains ${ }^{21}$. Déshumanisation des migrants à l'intérieur du camp qui se double d'une criminalisation à l'extérieur. Soustraits au regard des citoyens, transportés en fourgons cellulaires, menottés, enfermés dans des lieux qui présentent toutes les apparences des prisons, l'image que l'Etat assigne aux migrants est bien celle de criminels alors qu'il s'agit de personnes qui n'ont commis aucun autre délit que d'avoir franchi - ou tenté de franchir - des frontières sans les documents nécessaires. Image qui sert en retour à 
légitimer la politique d'enfermement et d'éloignement qui leur est appliquée. Quant à ceux qui expriment encore quelque doute, un troisième élément achève généralement de les convaincre : l'association récurrente des migrants à la menace terroriste depuis les attentats du 11 septembre 2001. Menace sérieuse à laquelle il convient de réagir par des moyens fermes : la boucle est alors bouclée.

Ensuite, deuxième trait commun qui découle du premier, ces camps sont le lieu de nombreuses violations des droits fondamentaux ${ }^{22}$. Parmi les droits et libertés violés, on peut relever : la liberté d'aller et venir, le droit à la vie privée et familiale, le droit de ne pas subir des traitements inhumains ou dégradants, le droit de demander l'asile ${ }^{23}$ ou encore les droits spécifiques aux mineurs. Ces droits et libertés sont pourtant tous garantis par des conventions internationales contraignantes pour les Etats membres. Il faut également souligner l'arbitraire et les discriminations qui règnent dans ces lieux. Tant dans leur fonctionnement quotidien - on pense notamment au flou qui entoure les sanctions disciplinaires - que, en amont ou en aval, dans la manière dont l'administration prend ses décisions. Il n'est d'ailleurs pas rare que des personnes chargées de l'aide juridique aux migrants et rompues au caractère technique des procédures se trouvent confrontées à des décisions totalement inexplicables.

Troisième trait commun à l'échelle européenne, cette fois dans la gestion des camps d'enfermement : une tendance, d'une part, à l'augmentation des capacités d'accueil et, d'autre part, au recours croissant à des compagnies privées. Les gouvernements italien, français et belge ont récemment annoncé leur intention d'augmenter les capacités des centres. Ces projets sont évidemment liés à la volonté affichée de «faire du chiffre » en matière d'éloignement du territoire. Le ministre de l'Intérieur français, Nicolas Sarkozy, n'avait-il pas annoncé son intention de doubler le nombre d'éloignements, ambition que son successeur s'est empressé de reprendre à son compte? Le ministre de l'Intérieur belge, Patrick Dewael, ne se déclare-t-il pas freiné dans ses ambitions d'opérations policières de grande envergure, en particulier dans les villes côtières et à Bruxelles, du fait du manque de places dans les centres fermés? Il est pourtant évident pour tout le monde, y compris pour les responsables politiques, que ces places supplémentaires ne changeront rien. Mais, comme on le devine - on y reviendra -, on se situe bien plus dans l'ordre symbolique que dans celui de l'efficacité. Il faut continuer de donner des gages de fermeté par rapport à la gravité d'une menace qu'on s'attache à entretenir. Quant à la privatisation de la gestion des lieux d'enfermement, elle est déjà largement en marche. En Grande-Bretagne, les six « centres d'expulsion " (removal centers) que compte le pays sont gérés par la société Group 4 sous le contrôle des services de l'immigration du ministère de l'Intérieur (Home Office). La même société procède également à des expulsions pour le compte de l'Etat. En Italie, en parallèle à l'adoption de la loi dite Bossi-Fini du 30 juillet 2002, le gouvernement de Silvio Berlusconi a étendu le pouvoir des préfets dans la gestion des CPTA en les autorisant à ouvrir de nouveaux centres et à signer des conventions avec des sociétés privées pour leur gestion.

Enfin et surtout, les camps d'enfermement d'étrangers en Europe présentent d'importants traits communs en termes d'(in)efficacité, d'objectifs annoncés et d'objectifs réels. Par efficacité des camps, on entend le nombre de personnes effectivement éloignées par rapport au nombre de personnes enfermées en vue de leur éloignement. En France, à partir des statistiques calculées sur dix ans (1992-2002), la Cimade relève qu'en moyenne $50 \%$ des étrangers retenus sont effectivement expulsés, 
avec une diminution progressive du taux d'éloignement passé de $61 \%$ en 1992-93 à $39,6 \%$ en 2002. Un travail sérieux reste à faire à l'échelle européenne - dans ce domaine sensible les chiffres sont difficiles à obtenir et les statistiques délicates à établir pour des observateurs extérieurs - mais on estime généralement que l'efficacité des centres d'éloignement se situe entre 40 et $60 \%$. Soit une efficacité assez faible, à mettre en rapport avec un coût opérationnel énorme. Là aussi le calcul du coût de l'enfermement des migrants reste à faire. Coût qui devrait englober d'autres éléments : la surveillance et les contrôles aux frontières; la construction et la maintenance des lieux d'enfermement; le traitement des dossiers administratifs et judiciaires; la nourriture et les soins donnés aux détenus; le transport et l'éloignement, depuis les voitures banalisées pour des reconduites à la frontière jusqu'à la location d'avions militaires. Avec une efficacité aussi réduite pour un coût aussi énorme, la portée réelle de ces camps se situe en réalité dans l'ordre symbolique. Il s'agit avant tout de mettre en scène et d'envoyer un double signal. D'une part, aux candidats-migrants à qui on espère signifier que la politique de lutte contre l'immigration clandestine (enfermementéloignement) sera poursuivie avec fermeté - c'est le fameux signal fort : «Voyez ce qui vous attend! »; d'autre part, aux opinions publiques internes à qui on espère démontrer que le problème est ainsi pris au sérieux: «Voyez, on s'en occupe!». Avec une conséquence importante pas assez soulignée: la fabrication et la reproduction de clandestins, constitués pour partie de ceux qui finissent par être libérés parce qu'inéloignables et de ceux qui, éloignés, reviennent dans le pays où ils ont leurs attaches ou un travail.

41 Mais au-delà d'une double portée symbolique, l'existence de ces camps rencontre aussi des objectifs moins avouables. D'abord, rendre moins visibles ceux qui gênent : mettre à l'écart des personnes dont le seul tort est de nous rappeler trop durement par leur présence l'incohérence de nos politiques migratoires et l'immensité du décalage entre notre niveau de vie et le leur. On touche ici au cœur du paradoxe puisque, dans le même temps, ces camps sont mis en avant comme gages de fermeté politique. Ensuite se débarrasser fort opportunément d'une charge politiquement et financièrement encombrante pour le trésor public: qu'une personne soit effectivement éloignée ou poussée à la clandestinité, il n'y a en effet plus d'aide sociale à payer.

\section{L'externalisation des politiques d'asile et d'immigration et la création de nouveaux camps}

La politique d'externalisation de l'Union européenne, en filigrane de nombreuses orientations de l'Union depuis plusieurs années, fait désormais partie des priorités de la toute nouvelle présidence luxembourgeoise. Réaffirmée dans le programme européen de La Haye ${ }^{24}$, elle consiste à faire reposer sur les pays d'origine et de transit des migrants le poids du traitement des demandes de migration et d'asile. Comment? Par le biais à la fois de "partenariats " pour développer et renforcer les contrôles aux frontières et par l'exportation des systèmes d'asile et d'immigration dans ces pays. Ainsi transposés, ces systèmes générateurs de camps, continueront à faire émerger des lieux d'enfermement ou de regroupement mais cette fois de l'autre côté de la frontière européenne.

En effet, pour gérer les populations migrantes vers l'Europe, les pays de transit devront obligatoirement mettre en place des lieux fermés, centres de rétention ou de détention, 
gérés par les autorités, afin de détenir pour identifier, détenir pour examiner une demande d'admission sur le territoire, détenir pour expulser. Le traitement des demandeurs d'asile passe aussi par la création de centres de réception. La notion de « capacity building» tend à développer dans les Etats à l'extérieur de l'Union, sur le modèle de ce qui a été mis en place dans les nouveaux Etats membres de l'Union européenne, des lieux de détention et de nombreux centres d'accueil pour demandeurs d'asile. En Pologne par exemple, ont été créés dans les 5 dernières années 13 centres de réception et une dizaine de centres de détention ${ }^{25}$.

Le Maroc a ainsi intégré dans sa toute nouvelle loi sur le séjour et l'entrée des étrangers la possibilité de créer des centres de rétention et des zones d'attente sur le modèle français ${ }^{26}$.

Mais l'exportation des camps pour étrangers ne s'arrête pas à ces camps formels, encadrés par le droit. On l'a vu, la lutte contre l'immigration clandestine et la fermeture des frontières génèrent les regroupements informels des populations migrantes vers l'Europe sur les territoires des Etats de transit. C'est déjà le cas avec le Maroc avec les camps de Gourougou et de Bel Younech, c'est le cas en Algérie et en Libye. Là encore, les droits fondamentaux des individus sont moins respectés qu'ailleurs. La politique d'externalisation qui consiste à renforcer les responsabilités des Etats " partenaires » dans la gestion de l'immigration favorise l'émergence de ces lieux dans des pays qui ne sont pas dotés de systèmes complets d'asile et de politique d'immigration.

Enfin pour clore le tableau, c'est dans la plus pure logique d'externalisation que le ministre de l'Intérieur allemand Otto Schily, soutenu par le chef du gouvernement italien Silvio Berlusconi, ont récemment remis sur le tapis européen l'idée de créer des camps ou «centres d'accueil» voire "portails d'immigration" pour gérer les populations migrantes en route vers l'Europe. Lancés par Tony Blair en 2003 puis abandonnés par ses partenaires européens au sommet de Thessalonique en juin 2003 car jugés dangereux et non viables, ces projets de créations de camps sont revenus à l'ordre du jour de l'agenda européen suite à l'affaire de Cap Anamur ${ }^{27}$ qui a occupé les médias de l'été 2004.

47 Ces lieux, que l'ancien commissaire européen à la Justice et Affaires Intérieures Antonio Vitorino refuse de désigner sous le terme trop évocateur de $"$ camps $»^{28}$, n'ont pas une fonction clairement établie. Si les propositions de 2003 avaient fait l'objet de quelques écrits et compléments de proposition apportés par la Commission européenne et le Haut Commissariat pour les Réfugiés, en 2004, seules des rumeurs par voie de presse, de surcroît parfois contradictoires, ont constitué la base des propositions germanoitaliennes : création de "portails d'immigration » en Afrique du Nord afin d'éviter la catastrophe humanitaire qui a lieu en mer Méditerranée, et qui a été provoquée par l'immigration clandestine. Par qui, comment et avec quelle finalité ces centres pourraient voir le jour, rien n'est moins clair. Ce qui apparaît cependant est cette volonté des Etats proposant de se débarrasser d'une population encombrante. Les récentes expulsions massives de l'Italie vers la Libye le confirment ${ }^{29}$.

L'Union européenne n'a pas nié la nécessité de traiter le problème et, bien que le sommet européen de Bruxelles des 4 et 5 novembre 2004 a été assez prudent pour ne pas aborder la question de ces centres externalisés, le Conseil, dans son programme de La Haye demande à la Commission européenne de mener " une autre étude [qui] devrait être menée en étroite consultation avec le HCR pour évaluer le bien fondé, le caractère opportun 
et la faisabilité d'un traitement commun des demandes d'asile en dehors du territoire de l'UE (...)». Ces centres ou camps pourraient donc avoir pour vocation de trier les demandeurs d'asile. Dans le flou le plus absolu et preuve d'un certain mélange des genres, le gouvernement français s'est opposé à la création de tels lieux lors de la réunion du G5 des 17 et 18 octobre 2004 arguant du fait que des camps d'attente génèrent les réseaux mafieux. En revanche, $M$. de Villepin, qui a reconnu l'urgence de la situation en Méditerranée, a accepté l'hypothèse de "points d'accueil » ou de "points de contact» en Afrique du Nord qui "donneraient la possibilité aux réfugiés arrivés du sud de l'Afrique de se renseigner sur leurs droits et de trouver aussi, éventuellement, les moyens de retourner chez eux si leurs demandes apparaissaient comme irrecevables (...) $)^{30}$.

On semble donc renoncer aux camps informels mais on prévoit des lieux d'accueil formels qui, si l'on pousse le raisonnement jusqu'au bout, sont encore dans une logique de contrôle des personnes qui "souhaitent retourner chez elles, après échec de leur demande »: centres de rétention? Camps de réfugiés? L'Italie n'a pas attendu ses homologues européens pour lancer un « projet pilote » avec la Libye pour la création de tels centres sur lesquels peu d'informations sont actuellement disponibles.

Ces efforts déployés par l'Union européenne pour se débarrasser de populations encombrantes ont pour conséquence inévitable la multiplication de lieux de détention ou de relégation des étrangers. Le camp est ainsi, sous ses aspects multiformes, un symbole d'une Europe en régression par rapport à ses valeurs et ses engagements internationaux

\section{NOTES}

1. La carte est publiée dans le présent numéro.

2. Une explication du choix sémantique du mot « camp » est disponible sur le site de Migreurop : http://pajol.eu.org/article675.html.

3. Communiqué de l'Afvic (Association des amis et familles des victimes de l'immigration clandestine) du 28 avril 2004.

4. Notamment le gouvernement belge. La France a soutenu cette position avant que la loi ne confère une existence officielle aux zones d'attente en 1992.

5. Le UK 1971 Immigration Act contient toutes les dispositions relatives à l'entrée irrégulière au Royaume-Uni et donne au Home Office le pouvoir de détenir les personnes qui ne respectent pas ces conditions.

6. Modifiée par la Loi Bossi-Fini de 2002 créant les Centri di Identificazione (CDI), autres lieux de détention pour demandeurs d'asile interceptés à la frontière.

7. Refugee Act, article 10 (2) (b).

8. Constats de la Cimade pour la France et du MRAX pour la Belgique.

9. La Cimade, service œcuménique d'entraide, bénéficie de la convention définie à l'Article $5 \mathrm{du}$ décret $n^{\circ}$ 2001-236 du 19 mars 2001.

10. En Grande-Bretagne, une association des visiteurs de «détenus pour questions d'immigration », Association of Visitors for Immigration Detainees (AVID), utilise le droit de visite 
pour assister les personnes et les aider à constituer leurs dossiers en les mettant en lien avec des avocats.

11. Voir en particulier pour la Grande-Bretagne les rapports de l'association «Bail for Immigration Detainees ".

12. Dans l'arrêt Chahal c. UK du 15 novembre 1996, la Cour a estimé que la durée de la détention était justifiée au regard de la complexité du dossier et qu'il n'y avait donc pas eu violation de l'article $51 \mathrm{f}$ ) de la Convention Européenne des Droits de l'Homme.

13. Loi Bossi-Fini $n^{\circ} 198 / 2002$ du 30 juillet 2002.

14. C'est le terme employé par deux parlementaires belges après une visite du centre fermé de Bruges en 1999. Voir Mayer N., «Les centres fermés : vue de l'intérieur », in Perrouty P.-A., La mise à l'écart de l'étranger - Centres fermés et expulsions, Bruxelles, Labor, 2004, p. 73 et suivantes.

15. Medici Senza Frontiere, "Rapporto suis Centri di permanenza temporanea e assistenza ", Missione Italia, janvier 2004.

16. Human Rights Watch, «L'autre visage des îles Canaries - violations des droits des migrants et des demandeurs d'asile », 2002.

17. Mission Internationale d'Enquête de la FIDH (Fédération Internationale des Ligues des Droits de l'Homme, "Enfermer les étrangers, dissuader les réfugiés : le contrôle des flux migratoires à Malte », $n^{\circ} 403$, septembre 2004.

18. Article paru dans Eleutherotypia le 29 août 2003.

19. Voir le rapport de la Cimade, «La situation alarmante des subsahariens en transit dans le nord du Maroc », 2004.

20. Sur la situation en Belgique, voir Mayer N., «Les centres fermés : vue de l'intérieur », op. cit.

21. Extraits d'une lettre signée par vingt-neuf détenus, juillet 2002.

22. Voyez Rodier C., «Les camps d'étrangers, dispositif clef de la politique d'immigration et d'asile de l'Union européenne ", in Perrouty P.-A., La mise à l'écart de l'étranger - Centres fermés et expulsions, op. cit., p. 32.

23. C'est le cas de nombreux migrants arraisonnés en mer ou échoués sur les côtes du sud de l'Italie ou de l'Espagne et qui sont souvent refoulés ou expulsés vers l'Afrique (Libye, Maroc, Tunisie) sans même avoir pu demander l'asile, en violation flagrante de la Convention de Genève.

24. Programme pour un espace de Liberté, Sécurité et Justice approuvé par le Conseil européen de Bruxelles les 4 et 5 novembre 2004.

25. Detention in Europe, JRS 2004.

26. Loi sur l'entrée et le séjour des Etrangers 02.03. Voir à ce sujet Belguendouz A., "Le Maroc, Vaste zone d'attente ", Plein Droit, $\mathrm{n}^{\circ}$ 57, juin 2003 ; ainsi que son article dans le présent numéro de Cultures \& Conflits.

27. Sur l'affaire de Cap Anamur voir le site Migreurop: http://www.migreurop.org/ rubrique146.html

28. Sur le sujet voir notamment «L'Europe financera des 'centres' de réfugiés en Afrique du Nord », Le Figaro, 2 octobre 2004.

29. Sur les expulsions vers la Libye, voir le site Migreurop: http://www.migreurop.org/ article659.html

30. Voir Le Monde, 19 octobre 2004. 


\section{RÉSUMÉS}

Lutter contre l'immigration clandestine est devenue une des obsessions majeures des Etats de l'Union européenne. Les camps d'enfermement d'étrangers qui constituent la partie la plus visible du processus se multiplient ainsi dans tous les Etats membres. Le réseau Migreurop a tenté de les recenser, d'en dresser une typologie et d'analyser les logiques à l'œuvre. L'article développe ces recherches, en soulignant d'une part l'hétérogénéité de formes et d'organisation de ces lieux de relégation et, d'autre part, des traits communs inquiétants. Lieux de déshumanisation des migrants et de multiples violations des droits fondamentaux, ces camps présentent une efficacité relativement faible au vu de leurs objectifs - pour un coût opérationnel énorme, de telle sorte que la raison de leur existence se situe largement dans l'ordre symbolique. L'Europe n'en reste pas là puisque l'heure est à l'exportation de ces méthodes et de ses conséquences.

Fighting against illegal immigration is one of the main obsessions of the European Union States. Detention camps, which are the visible part of the process, are thus mushrooming in all member States. The Migreurop Network has attempted to make an inventory of those camps, a typology, and to analyse the logics behind. The article develops on these researches highlighting the heterogeneity of form and organisation of these places, and underlying common worrying features. Where de-humanisation of migrants and violation of fundamental rights are taking place, these camps are relatively not efficient in the light of the objectives pursued and extremely costly. Their existence is therefore more justified by the symbol they represent. But Europe is currently in the process of exporting these methods and their consequences.

\section{INDEX}

Mots-clés : Immigration, camps, Europe, mise à l'écart des étrangers, migreurop

\section{AUTEURS}

\section{CAROLINE INTRAND}

Caroline Intrand est juriste à la Cimade à Paris, membre de l'équipe de coordination du service de la Défense des Etrangers Reconduits et spécialement chargée des questions européennes et du suivi des étrangers éloignés. Elle participe au réseau Migreurop.

\section{PIERRE-ARNAUD PERROUTY}

Pierre-Arnaud Perrouty est conseiller juridique au MRAX (Mouvement contre le racisme, l'antisémitisme et la xénophobie, Bruxelles) pour lequel il effectue régulièrement des visites dans les centres fermés. Il a coordonné l'ouvrage La mise à l'écart de l'étranger. Centres fermés et expulsions (Labor, 2004) et participe au réseau Migreurop. 